\title{
True factor analysis in medical imaging: Dealing with high-dimensional spaces
}

\author{
Alexei M. C. Machado \\ Pontifical Catholic University of Minas Gerais \\ Av. Dom Jose Gaspar, 500, Belo Horizonte, MG, Brazil \\ alexei@pucminas.br
}

\begin{abstract}
This article presents a new method for discovering hidden patterns in high-dimensional dataset resulting from image registration. It is based on true factor analysis, a statistical model that aims to find clusters of correlated variables. Applied to medical imaging, factor analysis can potentially identify regions that have anatomic significance and lend insight to knowledge discovery and morphometric investigations related to pathologies. Existent factor analytic methods require the computation of the sample covariance matrix and are thus limited to low-dimensional variable spaces. The proposed algorithm is able to compute the coefficients of the model without the need of the covariance matrix, expanding its spectrum of applications. The method's efficiency and effectiveness is demonstrated in a study of volumetric variability related to the Alzheimer's disease.
\end{abstract}

\section{Introduction}

The development of non-invasive medical imaging modalities opened a new perspective for in vivo studies, in which anatomical and functional aspects can be jointly observed. The representation of the overwhelming amount of data provided by imaging modalities and the extraction of relevant knowledge from the dataset became a crucial issue. The data should not only be represented in a manageable way, but also facilitate hypothesis-driven explorations of regional shape differences and lend deeper insight to morphometric investigation.

The comparison between the anatomies depicted by imaging modalities can be achieved by image registration, in which an image taken as a reference is brought into correspondence to the subjects' images in the study. Medical images may provide too much data for manual pointwise registration, what motivates the development of automatic methods that implement computer vision algorithms. The result of registration may, nevertheless, increase the amount of data to be analyzed to an intractable level. The information that reveals the association between variables is sparsely embedded in the covariance matrices, which can only be computed to low-dimensional variable spaces. For three-dimensional images that easily reach millions of variables, the computation of such matrices becomes an impossible task.

In this article we present a new method for knowledge discovery in medical imaging based on the factor analytic model. Factor analysis (FA) is an important multivariate statistical method in which the original observed variables are grouped into clusters that potentially share a common cause [11]. It is used, together with path analysis, to define structural equation models of the problem under investigation. In addition to the morphometric variables, clinical and demographic information can be considered in the analytic model and contribute to explore the relationship between regions in the image and pathologies or features of special interest. Factor analytic methods are based on the decomposition of the correlation matrix and this is a major limitation to the use of this method in medical imaging analysis. The method presented in this paper does not require the computation of the correlation matrix and is able to estimate all the coefficients needed to define the model. We demonstrate the exploratory potential of the new method in a study of morphologic differences in the neuroanatomy between patients diagnosed with Alzheimer's disease and normal controls.

\section{Image registration}

The purpose of image registration is to determine a correspondence between each voxel $\mathbf{q}$ in an image $I_{R}$, chosen as a reference, to a voxel $\mathbf{p}$ in another image $I_{T}$. The problem of matching can be stated as finding two functions $h$ and $g$ such that

$$
I_{R}(\mathbf{q})=g\left(I_{T}(h(\mathbf{q}))\right) .
$$

The intensity transformation function $g$ establishes a correspondence between the two image spectra. The spatial nonrigid transformation function $h$ maps corresponding voxels 
between the images:

$$
h(\mathbf{q})=\mathbf{q}+\mathbf{u}(\mathbf{q})=\mathbf{p}
$$

where $\mathbf{u}$ is a displacement field [9].

Non-rigid registration may require that the image volumes be described as continuous media with an associated constitutive model. Following the pioneer work of Bajcsy and collaborators [1] on the linear elasticity model, other models of spatial transformation have been developed, including modal matching [23], active shape models [3, 6], fluid mechanics [2] and active contours [4]. The displacement fields obtained from registration are detailed descriptions of the anatomy depicted in the images. The posterior analysis of these vector fields is able to reveal size and shape differences, regional volumetric variation, curvature and surface measurements, among others. For instance, when the reference image is warped to match a subject image, some regions may get enlarged and some may be reduced. It is possible to determine the amount of scaling applied to an infinitesimal volume around each voxel $\mathbf{q}$ of the reference image, by computing the Jacobian determinant of the spatial transformation [17]. The Jacobian determinant $J_{i}(\mathbf{q})$ is defined as the determinant of the gradient of the mapping function $\mathbf{q}+\mathbf{u}_{i}(\mathbf{q})$ :

$$
J_{i}(\mathbf{q})=\left|\nabla\left(\mathbf{q}+\mathbf{u}_{i}(\mathbf{q})\right)\right| .
$$

Since the Jacobians are computed from the results of image registration, which is basically a smooth transformation, it is expected that the values computed for neighbooring voxels be somehow correlated. The mapping of corresponding structures may also result in correlated values. Finally, different structures and regions of interest may present hidden patterns of correlation. Exploring these correlations is therefore an important source for knowledge discovery. The huge amount of data, nevertheless, is an obstacle for this task. Since the number of variables is much larger than the number of images in the study, the correlation matrices are not only singular, but also impossible to compute due to their processing and memory requirements. The Jacobians computed from the registration of a $128 \times 128 \times 128$ image, for example, result in a $2^{21} \times 2^{21}$ correlation matrix. The methods used to extract hidden patterns from this profuse amount of redundant information must therefore avoid any direct manipulation of the correlation matrix.

\section{The factor analytic model}

The purpose of FA is to explore the correlation among the variables of a problem, acting as a powerful method of data reduction, which makes it possible to manage the large amount of information obtained from image registration. A fundamental feature of FA is that, in addition to data reduction, it may favor data interpretation and knowledge discovery.

In the true factor analytic model, an $n$-dimensional set of observed variables, $\mathbf{z}=\left(z_{1}, \ldots, z_{n}\right)^{T}$, is represented as linear combinations of $m(m<<n)$ latent variables called commonfactors, $\mathbf{f}=\left(f_{1}, \ldots, f_{m}\right)^{T}$, and residual or unique factors, $\mathbf{y}=\left(y_{1}, \ldots, y_{n}\right)^{T}$, which account for the portion of $\mathbf{z}$ that is not common to other variables, including error:

$$
\mathbf{z}=\mathbf{A f}+\mathbf{U} \mathbf{y}
$$

where $\mathbf{A}=\left(\left(a_{11}, \ldots, a_{1 m}\right)^{T}, \ldots,\left(a_{n 1}, \ldots, a_{n m}\right)^{T}\right)^{T}$ is the loading matrix and $\mathbf{U}=\operatorname{diag}\left(u_{1}, \ldots, u_{n}\right)^{T}$ is the residual coefficients. The coefficients $a_{i j}$, called loadings, express the covariance between variable $z_{i}$ and factor $f_{j}$. Variables $\mathbf{z}$ are standardized, so that their expected values are 0 with variances equal to 1 . It is assumed that the expected values for the unique factors be 0 . Common and unique factors are not correlated, so that the covariance matrices equal the identity. In order for the factors to account for all the correlation among the variables, the covariances among unique factor terms and common factors are 0 .

Based on the relationship between observed variables, common and unique factors, the correlation matrix for the sample in the original space, $\mathbf{R}$, can be defined as

$$
\begin{aligned}
\mathbf{R} & =\operatorname{cov}(\mathbf{A f}+\mathbf{U} \mathbf{y}) \\
& =\operatorname{cov}(\mathbf{A f})+\operatorname{cov}(\mathbf{U} \mathbf{y}) \\
& =\mathbf{A} \operatorname{cov}(\mathbf{f}) \mathbf{A}^{T}+\mathbf{U} \operatorname{cov}(\mathbf{y}) \mathbf{U}^{T} \\
& =\mathbf{A} \mathbf{A}^{T}+\mathbf{U U}^{T} .
\end{aligned}
$$

From Eq. 2, the variance $r_{i}^{2}$ of a given variable $z_{i}$ can be decomposed into components due to the $m$ common factors, $a_{i 1}^{2}+\ldots+a_{i m}^{2}$, called the communality, and a specific variance $u_{i}^{2}$ :

$$
r_{i}^{2}=\sum_{j=1}^{m} a_{i j}^{2}+u_{i}^{2} .
$$

The number of factors, $m$, is a parameter to be determined either experimentally or by evaluating the statistical fit of the data in the model [11].

\section{Previous work}

The major objective of FA is to explore the inherent redundancy embedded in the covariance among the observed variables and investigate possible causal relationships with latent constructs. In this sense, it may be considered an appropriate alternative to simpler data reduction techniques such as the Principal Component Analysis, in which only the variance is considered [22]. On exploring the morphology of specific structures, one may be concerned with the 
relationship between regions of interest. FA may reveal aspects about the correlation between those regions and facilitates interpretation. Nonetheless, the use of FA in morphometry has been restricted to the representation of gross measurements and landmarks, in part due to the complexity of the covariance matrix computation, as exemplified in the works of Marcus [19] and Reyment and Jöreskog [21]. Stievenart et al. [24] also applied FA to study the corpus callosum, revealing factors clearly related to the curvature of specific parts of the callosal structure. Another relevant work on the factor analysis of the corpus callosum was presented by Deneberg et al. [5], in which the structure was divided into segments and the area was measured in order to correlate regions of interest to demographic information, such as gender and handiness. Machado et al. [16] applied FA to study the relationship among pointwise Jacobian determinants, obtained from automatic image registration. The method was able to partition the corpus callosum into substructures that were previously determined based on post mortem dissection of the fiber tract, proving the ability of FA to serve as a knowledge discovery tool. In all these works, the problem was made simpler by either using a lowdimensional variable space or by approximating the factor model, so that only common factors would be considered in the analysis

The critical problem in FA is the computation of the loading and residual coefficient, respectively represented by matrices $\mathbf{A}$ and $\mathbf{U}$. Many techniques have been proposed to determine these coefficients. The simplest one neglects $\mathbf{U}$ in Eq. 2 and factors $\mathbf{R}$ using spectral decomposition:

$$
\mathbf{R} \approx \hat{\mathbf{A}} \hat{\mathbf{A}}^{T}=\mathbf{Q} \mathbf{\Lambda} \mathbf{Q}^{T}=\left(\mathbf{Q} \boldsymbol{\Lambda}^{1 / 2}\right)\left(\mathbf{Q} \mathbf{\Lambda}^{1 / 2}\right)^{T},
$$

where $\boldsymbol{\Lambda}^{1 / 2}=\operatorname{diag}\left(\lambda_{1}^{1 / 2}, \ldots, \lambda_{n}^{1 / 2}\right)$ is the diagonal matrix with the square root of the eigenvalues of $\mathbf{R}$ and $\mathbf{Q}$ is the matrix of the corresponding eigenvectors. The loading matrix is thus estimated based on the sample covariance matrix as

$$
\hat{\mathbf{A}}=\mathbf{Q} \Lambda^{1 / 2} \text {. }
$$

The method described above for estimating the loading matrix A is very similar to Principal Component Analysis and is known as Principal Component Method [26]. By neglecting the specific variance matrix $\mathbf{U}$, the factor analysis of the covariance matrix $\mathbf{R}$ is performed by placing communalities in the diagonal elements. In this case, the recovered covariance matrix $\mathbf{A} \mathbf{A}^{T}$ have its off-diagonal elements affected.

A more precise method of evaluating the loadings in the true factor analytic model is the Principal Factor Method, in which the residuals are not necessarily small and cannot be neglected [11]. An initial estimate of $\mathbf{U}$ is subtracted from $\mathbf{R}$ and the result is eigendecomposed as

$$
\mathbf{R}-\mathbf{U U}^{T}=\mathbf{A} \mathbf{A}^{T}=\hat{\mathbf{Q}} \hat{\mathbf{\Lambda}} \hat{\mathbf{Q}}^{T}
$$

where $\hat{\mathbf{Q}}$ and $\hat{\Lambda}$ are respectively the eigenvectors and eigenvalues of $\mathbf{R}-\mathbf{U U}^{T}$. Loadings can thus be determined as $\mathbf{A}=\hat{\mathbf{Q}} \hat{\mathbf{\Lambda}}^{-1 / 2}$. The estimate of the residual coefficients is based on the inversed correlation matrix or requires, at least, the computation of $\mathbf{R}$ [20]. The principal factor method can be iterated to improve the estimates of the specific variances and achieve more precise loadings. The requirement of the correlation matrix is nevertheless a limitation to the method when dealing with high-dimensional variable spaces.

As an attempt to provide more robust and efficient solutions to the computation of loadings, Harman [11] proposed the Minres method (minimmum residuals) in which a solution for $\mathbf{A}$ is searched so as to minimize only the offdiagonal residuals of the difference between the observed correlation matrix $\mathbf{R}$ and the recovered correlations in the solution $\mathbf{A A}^{T}$ :

$$
\min _{A}\left\|[\mathbf{R}-\mathbf{I}]-\left[\mathbf{A} \mathbf{A}^{T}-\operatorname{diag}\left(\mathbf{A} \mathbf{A}^{T}\right)\right]\right\|,
$$

where $\mathbf{I}$ is the identity matrix. Also of special interest is the Maximum-Likelihood method, proposed by Lawley [15] and enhanced by Jöreskog and collaborators [12, 14, 13]. In this method, the loadings are computed and the goodness of fit of the model assessed simultaneously. The method minimizes the function

$\log \left|\mathbf{A} \mathbf{A}^{T}+\mathbf{U U}^{T}\right|+\operatorname{tr}\left(\mathbf{R}\left(\mathbf{A} \mathbf{A}^{T}+\mathbf{U U}^{T}\right)^{-1}\right)-\log |\mathbf{R}|-n$.

Methods based on minimization compare the observed correlations to the solution and are therefore also limited by the dimensionality of the problem.

\section{Methods}

The proposed method is an iterative procedure that simultaneously computes the loadings and the residual coefficients, without the need of the covariance matrix. From the fundamental equation of the true factor model (Eq. 2), matrix $\mathbf{A}$ is first estimated by neglecting the residual term:

$$
\mathbf{R} \approx \mathbf{A A}^{T}
$$

Instead of decomposing the correlation matrix into its eigenvectors, as performed in the principal factor method (Eq. 4), we apply singular value decomposition to the sample itself. Singular value theory states that any $n \times N$ matrix $\mathbf{Z}$ can be decomposed as:

$$
\mathbf{Z}=\mathbf{Q D W}^{T}
$$

where $\mathbf{Q}$ is an $n \times n$ matrix whose columns are the eigenvectors of $\mathbf{Z} \mathbf{Z}^{T} / N, \mathbf{W}$ is an $N \times N$ matrix whose columns are the eigenvectors of $\mathbf{Z}^{T} \mathbf{Z} / N$ and $\mathbf{D}=\boldsymbol{\Lambda}^{1 / 2}$ is an $n \times N$ matrix whose values in the diagonal are the square roots of 
the eigenvalues of both $\mathbf{Z} \mathbf{Z}^{T} / N$ and $\mathbf{Z}^{T} \mathbf{Z} / N$. Since $\mathbf{Q}$ is orthogonal, we have that

$$
\begin{array}{ll}
\mathbf{Q} \Lambda^{1 / 2} \mathbf{W}^{T} & =\mathbf{Z} \\
\mathbf{Q} \Lambda^{1 / 2} \mathbf{W}^{T} \mathbf{W} & =\mathbf{Z W} \\
\mathbf{Q} \Lambda^{1 / 2} \boldsymbol{\Lambda}^{-1 / 2} & =\mathbf{Z W} \boldsymbol{\Lambda}^{-1 / 2} \\
\mathbf{Q} & =\mathbf{Z W} \boldsymbol{\Lambda}^{-1 / 2}
\end{array}
$$

From Eq. 4 and 5, the first estimate of the loading matrix is

$$
\mathbf{A} \approx \mathbf{Q} \mathbf{\Lambda}^{1 / 2}=\mathbf{Z W} .
$$

In order to compute the estimate of the residual coefficients $\mathbf{U}$, we next apply the results obtained for the common loadings in Eq. 6 to the true factor analytic model (Eq. 1) and estimate the sample factor scores $\mathbf{F}$ following the approach described in [18]. This is achieved by globally minimizing the residual terms in Eq. 1, so that they account for the part of the variables that are really unique and cannot be represented by the common factors. From the factor scores, the residuals are computed as

$$
\mathbf{U Y} \approx \mathbf{Z}-\mathbf{A F}
$$

and the residual coefficients $\mathbf{U}$ determined by taking the covariance of the residual terms:

$$
\operatorname{cov}(\mathbf{U Y})=\mathbf{U}
$$

since the covariance of $\mathbf{Y}$ is the identity.

We now compute the next estimate of A. From Eq. 2, the loading matrix $\mathbf{A}$ could be determined as

$$
\mathbf{A} \mathbf{A}^{T}=\mathbf{R}-\mathbf{U U}^{T},
$$

i.e., by replacing the communalities in the diagonal of the correlation matrix and eigendecomposing the result. In this case, we would require the sample correlation matrix $\mathbf{R}$ which is known to be intractable. Instead, since $\mathbf{R}-\mathbf{U U}^{T}=\operatorname{cov}(\mathbf{Z}-\mathbf{U Y})$, the singular value decomposition is applied to $\mathbf{Z}-\mathbf{U Y}$, analogously as it was to $\mathbf{Z}$ in Eq. 5, yielding to the eigenvectors $\hat{\mathbf{Q}}$ and eigenvalues $\hat{\boldsymbol{\Lambda}}$ of $\mathbf{R}-\mathbf{U U}^{T}$ :

$$
\hat{\mathbf{Q}}=(\mathbf{Z}-\mathbf{U Y}) \hat{\mathbf{W}} \hat{\mathbf{\Lambda}}^{-1 / 2},
$$

where $\hat{\mathbf{W}}$ is the matrix whose columns are the eigenvectors of $(\mathbf{Z}-\mathbf{U Y})^{T}(\mathbf{Z}-\mathbf{U Y}) / N$. Finally, the new estimate of A can be computed from

$$
\mathbf{A A}^{T}=\operatorname{cov}(\mathbf{Z}-\mathbf{U Y})=\hat{\mathbf{Q}} \hat{\mathbf{\Lambda}} \hat{\mathbf{Q}}^{T}
$$

, as

$$
\mathbf{A}=\hat{\mathbf{Q}} \hat{\mathbf{\Lambda}}^{-1 / 2}=(\mathbf{Z}-\mathbf{U Y}) \hat{\mathbf{W}} \text {. }
$$

The process should be repeated from Eq. 7 to 8, until no more substantial changes in the loadings occur. The patterns revealed by the correlations between observed and latent variables, expressed by the loadings, can now be explored.

\section{A case study on the Alzheimer's disease}

The ability and efficiency of the proposed algorithm is illustrated in a case study on the Alzheimer's disease (AD) and its manifestations on the neuroanatomy of patients, compared to normal elderly controls. The dataset consists of 24 MRI image volumes from which 12 are diagnosed with $\mathrm{AD}$ (age $=70.8 \pm 8.5)$ and the remaining are matched controls (age $=68.5 \pm 9.4)$ [10]. The T1-weighted spoiled echo images were acquired on a GE 1.5 Tesla instrument (flip angle $=30^{\circ}, \mathrm{TR}=35 \mathrm{~ms}, \mathrm{TE}=6 \mathrm{~ms}, 1.3 \mathrm{~mm}$ slice thickness, in-plane resolution of $0.9 \times 0.9 \mathrm{~mm}$ ), and stored as $182 \times 218 \times 182$ volumes.

The brain volumes were registered to an average T1 template computed from 305 volumes [7], using SPM99 [8] with 12-parameter affine registration and non-linear registration with 12 iterations and $7 \times 8 \times 7$ basis function. The displacement fields obtained from image registration were the basis for the computation of the Jacobian determinants. Data matrix $\mathbf{Z}$ was formed by the determinant of the Jacobian matrix at each of the 2.1 million voxels of the stripped brain, together with the diagnosis, and used as input to FA. After each estimate of the loading matrix $\mathbf{A}$, it was rotated in order to maximize the variance of the squared loadings in each column, so that each variable presented high loading for fewer factors (quartimax algorithm) [21].

The proposed algorithm for estimating the loadings took 5 iterations to converge to a solution in which the absolute difference between the loadings in 2 consecutive iterations was smaller than 0.01 . The method revealed 23 factors associated to regional size variation, from which 4 was found to be highly correlated to the disease (absolute loadings greater than 0.30): factor 1 is associated with the overall cortical tissue at cerebrum and cerebellum; factor 2 is related to the ventricle enlargement; factor 9 encompasses cortical regions in the frontal lobe and right insular gyrus; and factor 21, related to the right occipital gyrus. The effects of cortical degeneration and ventricular enlargement are depicted in Fig. 1 and 2, respectively. The figures show a sequence of horizontal slices, from the inferior to the superior view of the brain, in which the white regions correspond to the voxels related to each factor. A summary of the results is described in Table 1. The findings are in accordance to the anatomical differences between patients and controls, reported in the literature [25], that reveal cortical degeneration and proportional enlargement of the ventricles associated to the disease. 


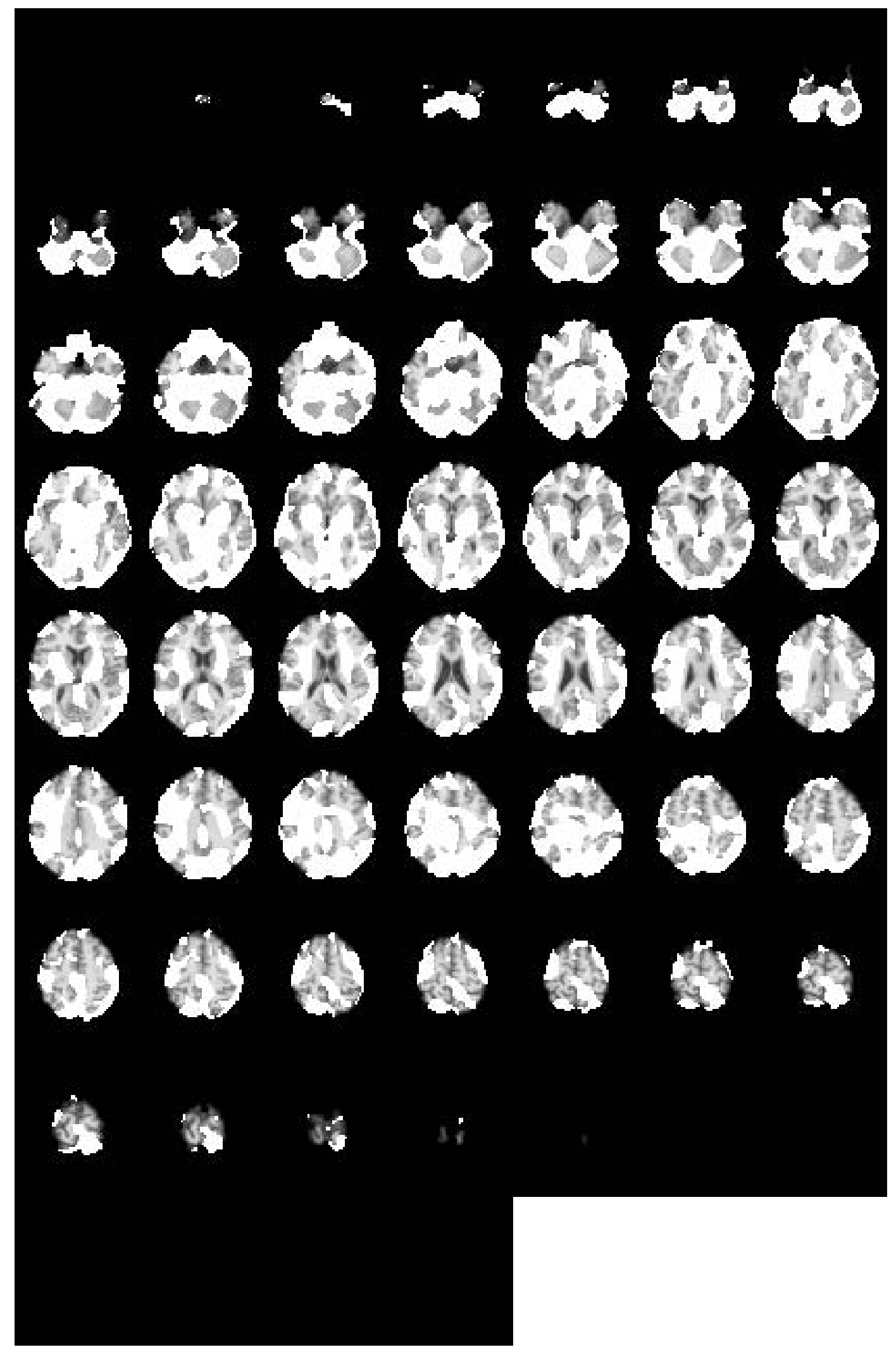

Figure 1. Regions of the brain associated to factor 0 whose size variation present high correlation to Alzheimer's disease (in white). The analysis reveals that general cortical degeneration is a significant feature related to the pathology. 


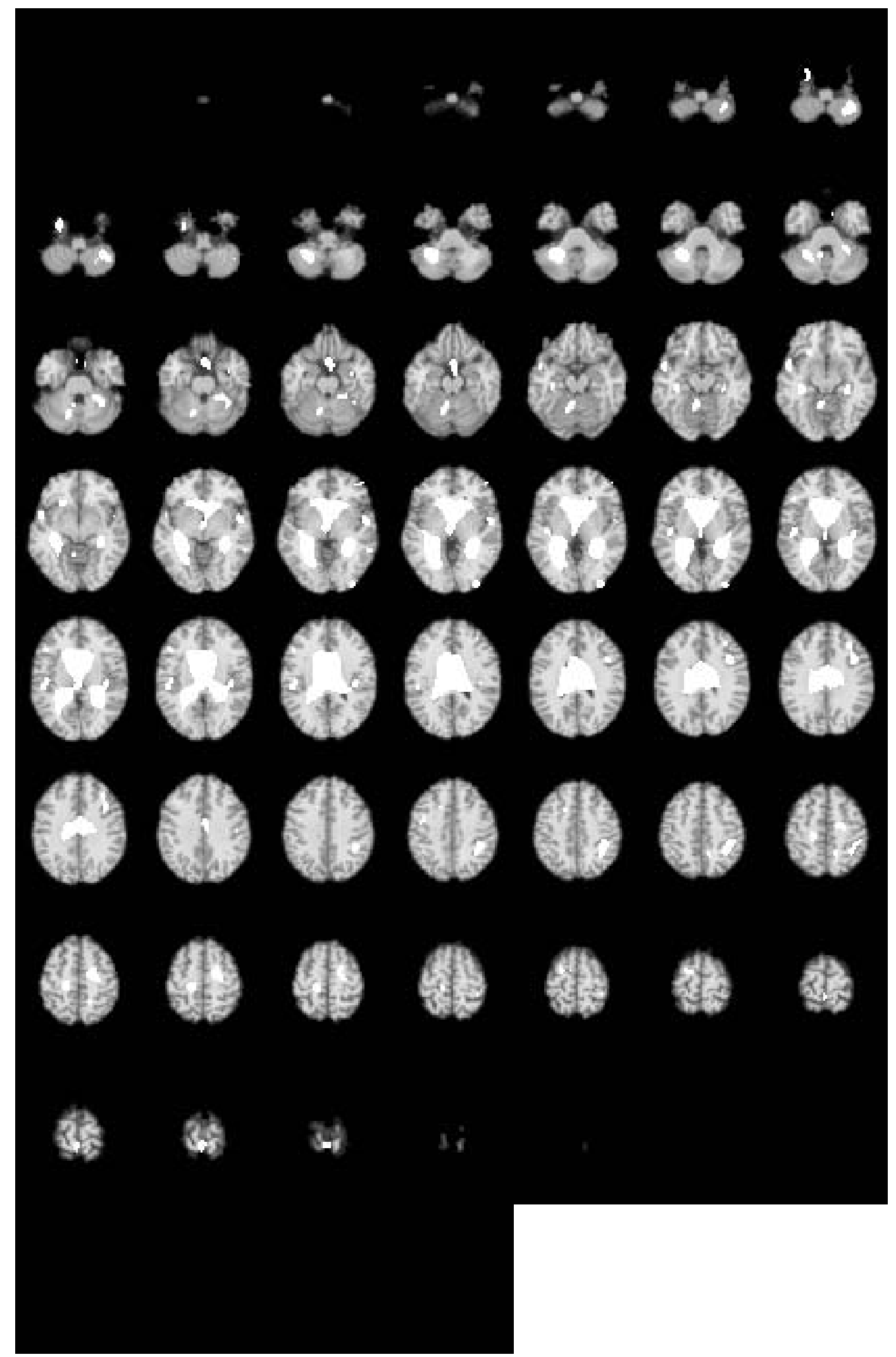

Figure 2. Regions of the brain associated to factor 1 whose size variation present high correlation to Alzheimer's disease (in white). The analysis reveals that general ventricular enlargement is a significant feature related to the pathology. 


\section{Conclusion}

A novel method for the estimation of loadings in true factor analysis was presented. It is able to compute common and residual coefficients without the need of the correlation matrix, which is intractable in high-dimensional variable space such the ones found in voxel-based registration problems. The algorithm iteratively computes better estimates of the correlations between observed and latent variables, without neglecting residual terms. The ability of the method to act as a knowledge discovery tool was demonstrated in a case study of the Alzheimer's disease. The factors revealed in the analysis are associated to regions of interest that are affected by the degenerative process related to the pathology.

The application of FA to high-dimensional representations of the anatomy is particularly advantageous, since the method facilitates the interpretation of the results. The factors can be visually identified as regions that embed strong correlation. Even when applied to small datasets, FA was able to provide results that are in accordance to clinical findings and additionally provided information about the correlation among morphological variables in a region of interest.

Acknowledgments - This work was partially supported by CNPq-Brazil. The author is grateful to the University of Pennsylvania for sharing the AD data.

\section{References}

[1] R. Bajcsy and S. Kovacic. Multiresolution elastic matching. Computer Vision, Graphics and Image Processing, 46:1-21, 1989.

[2] G. Christensen, R. Rabbitt, and M. Miller. Deformable templates using large deformation kinematics. IEEE Transactions on Image Processing, 5(10):1435-1447, 1996.

[3] T. Cootes, A. Hill, C. J. Taylor, and J. Haslam. Use of active shape models for locating structures in medical images. Image and Vision Computing, 12(6):355-365, 1994.

[4] C. Davatzikos and J. Prince. An active contour model for mapping the cortex. IEEE Transactions on Medical Imaging, 14:65-80, 1995.

[5] V. H. Denenberg, A. Kertesz, and P. E. Cowell. A factor analysis of the human's corpus callosum. Brain Research, 548:126-132, 1991.

[6] N. Duta and M. Sonka. Segmentation and interpretation of mr brain images: An improved active shape model. IEEE Transactions on Medical Imaging, 17(6):1049-1062, 1995.

[7] A. Evans, D. Collins, E. Brown, R. Kelly, and T. Petters. A 3D statistical neuroanatomical models for 305 MRI volumes. In IEEE Nuclear Science Symposium/Medical Imaging Conference, pages 1813-1817, 1993.

[8] R. Frackowiak, K. Friston, C. Frith, R. Dolan, and J. Mazziotta. Human Brain Function. Academic press, San Diego, 1997.
[9] J. C. Gee. On matching brain volumes. Pattern Recognition, 32:99-111, 1999.

[10] M. Grossman, C. McMillan, P. Moore, L. Ding, G. Glosser, M. Work, and J. Gee. What's in a name: voxel-based morphometric analyses of MRI and naming difficulty in alzheimer's disease, frontotemporal dementia and corticobasal degeneration. Brain, 127:1-22, 2004.

[11] H. Harman. Modern Factor Analysis. University of Chicago Press, Chicago, 1976.

[12] K. Jöreskog. Some contributions to maximum likelihood factor analysis. Psychometrika, 32:443-482, 1967.

[13] K. Jöreskog and A. S. Goldberger. Factor analysis by generalized least squares. Psychometrika, 37:243-260, 1968.

[14] K. Jöreskog and D. N. Lawley. New metrods in maximum likelihood factor analysis. British Journal of Mathematical and Statistical Psychology, 21:85-96, 1968.

[15] D. N. lawley. The estimation of factor loadings by the method of maximum likelihood. Proceedings of the Royal Society of Edinburgh, 60:64-82, 1940.

[16] A. Machado, J. C. Gee, and M. Campos. Exploratory factor analysis in morphometry. Lecture Notes in Computer Science, 1679:378-385, 1999.

[17] A. Machado, J. C. Gee, and M. Campos. Structural shape characterization via exploratory factor analysis. Artificial Intelligence in Medicine, 30:97-118, 2004.

[18] A. Machado, J. C. Gee, and M. Campos. Visual data mining for modeling prior distributions in morphometry. IEEE Signal Processing Magazine, 21(3):20-27, 2004.

[19] L. Marcus. Traditional Morphometrics. In F. J. Rohlf and F. L. Bookstein, editors, Proceedings of the Michigan Morphometrics Workshop, pages 77-122, Ann Arbor, 1990. The University of Michigan Museum of Zoology.

[20] A. Rencher. Methods of Multivariate Analysis. John Wiley \& Sons, New York, 1995.

[21] R. Reyment and K. Jöreskog. Applied Factor Analysis in the Natural Sciences. Cambridge University Press, Cambridge, 1996.

[22] S. Roweis and Z. Ghahramani. A unifying review of linear gaussian models. Neural Computation, 11(2):305-345, 1999.

[23] S. Sclaroff and A. Pentland. Modal matching for correspondence and recognition. IEEE Transactions on Pattern Analysis and Machine Intelligence, 17(6):545-561, 1995.

[24] J. L. Stievenart, M. T. Iba-Zizen, A. Tourbah, A. Lopez, M. Thibierge, A. Abanou, and A. Canabis. Minimal surface: A useful paradigm to describe the deeper part of the corpus callosum? Brain Research Bulletin, 44(2):117-124, 1997.

[25] P. M. Thompson, J. Moussai, S. Zohoori, A. Goldkorn, A. Khan, M. S. Mega, G. Small, J. Cummings, and A. W. Toga. Cortical variability and assymetry in normal aging and Alzheimer's disease. Cerebral Cortex, 8:492-509, 1998.

[26] G. H. Thomson. Hotelling's method modified to give Spearman's g. Journal of Educational Psychology, 25:366-374, 1934. 
Table 1. Summary of the factor analysis results. For each factor $f_{i}$, the table shows, from left to right, the number of voxels for which the factor presents the highest loading values, the loading of the factor to diagnosis and a description of the major regions associated to the factor, within the brain.

\begin{tabular}{|c|c|c|c|}
\hline$f$ & volume & loading & description \\
\hline 1 & 1077759 & 0.32 & $\begin{array}{l}\text { overall cortical region } \\
\text { at cerebrum and cerebellum }\end{array}$ \\
\hline 2 & 149742 & -0.53 & $\begin{array}{l}\text { ventricles and regions in } \\
\text { cerebellar white matter }\end{array}$ \\
\hline 3 & 54756 & 0.02 & fiber tracts from the splenium \\
\hline 4 & 34317 & 0.02 & $\begin{array}{l}\text { anterior medial part of } \\
\text { temporal lobes }\end{array}$ \\
\hline 5 & 26568 & 0.10 & isolated regions of the cortex \\
\hline 6 & 28458 & 0.06 & right superior frontal cortex \\
\hline 7 & 27135 & -0.05 & left insular gyrus \\
\hline 8 & 32292 & -0.09 & right frontal cingulated gyrus \\
\hline 9 & 13230 & 0.39 & $\begin{array}{l}\text { cortical regions in the frontal } \\
\text { lobe and right insular gyrus }\end{array}$ \\
\hline 10 & 29106 & 0.05 & left parietal cortex \\
\hline 11 & 15822 & 0.13 & right anterior frontal cortex \\
\hline 12 & 18954 & 0.13 & right posterior frontal cortex \\
\hline 13 & 22869 & -0.15 & left temporal white matter \\
\hline 14 & 15714 & -0.07 & left parietal cortex \\
\hline 15 & 23112 & -0.04 & left superior parietal cortex \\
\hline 16 & 21897 & -0.25 & right cerebellar white matter \\
\hline 17 & 6345 & 0.05 & $\begin{array}{l}\text { left inferior parietal } \\
\text { white matter }\end{array}$ \\
\hline 18 & 13716 & 0.11 & occipital cingulated gyrus \\
\hline 19 & 6993 & -0.24 & left temporal cortex \\
\hline 20 & 9666 & -0.15 & isolated regions of the cortex \\
\hline 21 & 9045 & 0.39 & right occipital gyrus \\
\hline 22 & 4563 & -0.03 & isolated regions of the cortex \\
\hline 23 & 864 & -0.24 & isolated regions of the cortex \\
\hline
\end{tabular}

